\title{
Threatened Freshwater Fishes of the United States
}

\author{
Robert Rush Miller ${ }^{1}$ \\ Museum of Zoology, The University of Michigan \\ Ann Arbor, Michigan 48104
}

\begin{abstract}
Threatened, native freshwater fishes are listed for 49 of the $50 \mathrm{U}$. S. States, the first such compilation. Over 300 kinds are included in a formal classification, cross-indexed to states (Table 1), followed by state lists and the status of each fish, whether rare, endangered, depleted, or undetermined. The concern for native fishes and the important factors responsible for threats to their existence are briefly outlined. Although the lists vary from those based on extensive recent state surveys to others in which current information is sparse, publication is expected to enhance the chances for survival through protective legislation (already enacted by a number of states) and stronger concern for such natural resources.
\end{abstract}

\section{INTRODUCTION}

It is only within the past decade, principally since 1963 , that serious threats to the survival of many of our unique native fishes have become widely recognized. Committees concerned with vanishing U. S. wildlife have been established within scientific societies as well as by the states and the federal government. On a global basis, action has been taken by the Survival Service Commission of the International Union for Conservation of Nature and Natural Resources, based in Morges, Switzerland. This has resulted in the preparation of lists of fishes that are or may be in trouble. The Endangered Species Preservation Act of 1966 marked the first concerted effort to protect our native fauna.

The present effort is the result of the activities of both the Conservation Committee of the American Society of Ichthyologists and Herpetologists (ASIH) and the Endangered Species Committee of the American Fisheries Society (AFS), whose members have worked for several years to compile lists of threatened native fishes of the United States. We have also utilized the Red Book of Rare and Endangered Fish and Wildlife of the United States (1968, revised edition in preparation), published by the U. S. Department of the Interior, and the IUCN Red Data Book (Vol. 4, Pisces, 1969). Coordination of this project was made easier because the writer has served as chairman of the AFS, ASIH, and IUCN

\footnotetext{
${ }^{1}$ Chairman, Endangered Species Committee, American Fisheries Society.
}

committees during the period that the information presented below was being prepared.

Since the late 1960's, particularly in the West but also in the East, states have been enacting legislation and enforcing protection of endangered species and subspecies as well as maintaining natural habitats and creating refugia for some of the more critically threatened fishes (see, for example, Miller and Pister, 1971). This type of effort must be extended into every state. In June, 1971, a bill was introduced into Congress to establish a Desert Pupfish National Monument to save the threatened pupfishes living east of Death Valley (Deacon and Bunnell, 1970). Such activities have evolved through efforts at private, state and federal levels, including the news media, and are increasing as we advance through the "environmental decade."

This is the first attempt to list the native threatened fishes for each of the $50 \mathrm{U}$. S. States; all presented lists except Rhode Island, which has no threatened fishes (John M. Cronan, pers. com., June, 1971). A total of 305 kinds are threatened in the United States (Table 1). Rare and endangered fishes of Canada have been treated by McAllister (1970), with a revision now in preparation.

Recognition that species or subspecies of fishes are threatened locally or nationally constitutes a primary step that can lead to the perpetuation of many of them. Without up-to-date lists of those being threatened, however, appropriate action cannot be taken. Although the status of many fishes here treated is not clear and the completeness of 
the state listings varies greatly, the present account is believed to be a reasonable current assessment (as of July, 1971). For a few states, the lists are complete; for many there is urgent need for ongoing surveys; for some, current information is almost nil. Despite these shortcomings, it is felt that publication of these lists will be of value in stimulating protective legislation for forms known to be under threat as well as initiating greater concern for all native wildlife. The states have the direct responsibility for managing and regulating the taking of fishes within their borders, and they must provide the required legal protection and enforcement for threatened species.

Some of the factors responsible for diminution of our fish faunas have been pollution (industrial, agricultural, and domestic, including toxic chemicals and pesticides), excessive damming of rivers (producing lentic versus lotic habitats), deforestation and overgrazing, channelization, excessive removal of ground water, and introduction of exotic species (especially predators or those with broader ecological tolerances than native forms). Exotics may also transmit parasites and diseases. The role of such factors in depleting, decimating, or exterminating fishes has been treated by Deacon and Bunnell (1970), Hubbs (1963), Lachner et al. (1970), Larimore and Smith (1963), McDowall (1968), Miller (1961), Mills et al. (1966), Minckly and Deacon (1968), Myers (1965), Scott (1963), Smith (1971), Trautman (1957), and others.

There is an urgent need to maintain diversity of life so as to provide for the variety of wildlife needed for recreation, commerce, and scientific study. It will take money and the efforts of dedicated and skilled people, combining their talents and working through legal channels, to produce effective programs for halting the depletion of fish resources.

The following served as members of the Endangered Species Committee, 1969-1971, and participated actively in this endeavor: Donald Andriano, James G. Armstrong, III, Howard M. Bassett, Carl E. Bond, Robert L. Borovicka, Alex Calhoun, Leonard Fisk, James R. Gammon, Richard H. Goodyear, George D.
Holton, Clark Hubbs, Robert A. Kuehne, A. C. Lopinot, John S. Ramsey, and William B. Smith.

The arrangement of families and the usage of common and scientific names, with only a few exceptions, are those recommended by the Commiittee on Names of Fishes of the American Fisheries Society (Bailey et al., 1970). A reference is provided for species described subsequent to that contribution. All lists were submitted for approval by the states prior to publication.

The definitions employed for determining the status of each fish are those developed by the IUCN, as given below. One category, "peripheral" fishes, that might have been used in assessing status, concerns those fishes common elsewhere but rare in a state. Such species have been listed as rare for some states but not for all. These populations, often disjunct, merit inclusion because isolated gene pools may have practical and scientific value.

Endangered: Actively threatened with extinction. Continued survival unlikely without the implementation of special protective measures.

Rare: Not under immediate threat of extinction, but occurring in such small numbers and/or in such a restricted or specialized habitat that it could quickly disappear. Requires careful watching.

Depleted: Although still occurring in numbers adequate for survival, the species has been heavily depleted and continues to decline at a rate substantially greater than can be sustained.

Indeterminate: Apparently threatened but insufficient data currently available on which to base a reliable assessment of status.

The factor common to all threatened species is not deterioration of status, but vulnerability to extinction in the foreseeable future. Thus populations of many "rare" species are relatively stable, and a "depleted" species may be retained in the lists for a period, after its populations have started to increase as a result of better management. Numerous factors, both genetic and environmental, affect the 
probability of a species' survival if it is rare or subjected to pressure. Although the status of many species leaves little doubt as to whether or not they are threatened, decisions on borderline cases must, of necessity, be made on their individual merits.

TABLE 1.-Threatened fishes of the United States. An asterisk indicates forms nationally recognized as endangered; the states listing each fish are given.

Lampreys, family Petromyzontidae

Ohio lamprey, Ichthyomyzon bdellium (Jordan). Md.

Chestnut lamprey, Ichthyomyzon castaneus Girard. Kan, Neb.

Southern brook lamprey, Ichthyomyzon gagei Hubbs and Trautman. Mo.

Allegheny brook lamprey, Ichthyomyzon greeleyi Hubbs and Trautman. Penn.

Silver lamprey, Ichthyomyzon unicuspis Hubbs and Trautman. Neb, S Dak, W Va.

American brook lamprey, Lampetra lamottei (Lesueur). Conn, Mass, Mo.

Pit-Klamath brook lamprey, Lampetra lethophaga Hubbs (1971). Ore.

Sturgeons, family Acipenseridae

* Shortnose sturgeon, Acipenser brevirostrum Lesueur. Conn, Del, Md, Mass, N J, N Y, Penn.

Lake sturgeon, Acipenser fulvescens Rafinesque. Ala, Ga, Ia, Mich, Minn, Mo, Neb, N Y, Ohio, Penn, S Dak, Vt, W Va.

Atlantic sturgeon, Acipenser oxyrhynchus Mitchill. Ala, Conn, Del, Ga, Me, Md, Mass, Miss, $\mathbf{N}$ Hamp, N J, Penn, S Car.

White sturgeon, Acipenser transmontanus Richardson. Ida, Mont.

Pallid sturgeon, Scaphirhynchus albus (Forbes and Richardson). Ia, Kan, Ky, La, Mo, Mont, Neb, N Dak, S Dak.

Shovelnose sturgeon, Scaphirhynchus platorynchus (Rafinesque). Ala, Ky, Minn, Miss, Okla, S Dak, W Va, Wyo.

Paddlefishes, family Polyodontidae

Paddlefish, Polyodon spathula (Walbaum). Md, Minn, Penn, W Va, Wisc.

Gars, family Lepisosteidae

Longnose gar, Lepisosteus osseus (Linnaeus). Del, S Dak.

Shortnose gar, Lepisosteus platostomus Rafinesque. Mont.

Alligator gar, Lepisosteus spatula Lacépède. Mo.

Bowfins, family Amiidae

Bowfin, Amia calva Linnaeus. Md, Penn, S Dak.

Freshwater eels, family Anguillidae

American eel, Anguilla rostrata (Lesueur). S Dak, Wisc.

Herrings, family Clupeidae

Alabama shad, Alosa alabamae Jordan and Evermann. Mo.

Skipjack herring, Alosa chrysochloris (Rafinesque). S Dak.

American shad, Alosa sapidissima (Wilson). Del, N J, N Y.

Mooneyes, family Hiodontidae

Goldeye, Hiodon alosoides (Rafinesque). Wisc, Wyo.
Mooneye, Hiodon tergisus Lesueur. Mich, Neb, Ohio, S Dak, Vt, W Va.

Trouts, family Salmonidae

*Longjaw cisco, Coregonus alpenae (Koelz). Mich, Wisc.

Cisco or lake herring, Coregonus artedii Lesueur. Ohio, Penn.

Lake whitefish, Coregonus clupeaformis (Mitchill). N Y, Ohio, Penn.

Kiyi, Coregonus kiyi (Koelz). Mich, Wisc.

Blackfin cisco, Coregonus nigripinnis (Gill). Wisc.

Shortnose cisco, Coregonus reighardi (Koelz). Wisc.

Shortjaw cisco, Coregonus zenithicus (Jordan and Evermann). Mich, Wisc.

Bear Lake whitefish, Prosopium abyssicola (Sny. der). Ida, Utah.

Round whitefish, Prosopium cylindraceum (Pallas). Conn, N Y.

Bonneville cisco, Prosopium gemmiferum (Snyder). Ida, Utah.

Bonneville whitefish, Prosopium spilonotus (Snyder). Ida, Utah.

Little Kern golden trout, Salmo aguabonita whitei Evermann. Cahif.

* Lahontan cutthroat trout, Salmo clarki henshawi Gill and Jordan. Calif.

Colorado cutthroat trout, Salmo clarki pleuriticus Cope. Colo, Wyo.

*Piute cutthroat trout, Salmo clarki seleniris Sny. der. Calif.

*Greenback cutthroat trout, Salmo clarki stomias Cope. Colo.

Rio Grande cutthroat trout, Salmo clarki virginalis (Girard). Colo, N Mex.

Alvord cutthroat trout, Salmo clarki subsp. Ore.

Westslope cutthroat trout, Salmo clarki subsp. Mont.

Eastslope cutthroat trout, Salmo clarki subsp. Mont.

Snake River cutthroat trout, Salmo clarki subsp. Wyo.

Snake Valley cutthroat trout, Salmo clarki subsp. Nev.

*Gila trout, Salmo gilae Miller. N Mex.

Atlantic salmon, Salmo salar Linnaeus. Conn, Me, Mass, N Hamp, N Y, Vt.

* Arizona trout, Salmo sp. Ariz. (see Miller, 1972).

Redband trout, Salmo sp. Calif, Ore.

Sunapee trout, Salvelinus alpinus aureolus Bean. Me, N Hamp.

Blueback trout, Salvelinus alpinus oquassa (Girard). Me.

Brook trout, Salvelinus fontinalis (Mitchill). Ohio.

Dolly Varden, Salvelinus malma (Walbaum). Ore.

Lake trout, Salvelinus namaycush (Walbaum). Ohio.

Angayukaksurak char, Salvelinus sp. Alaska.

Arctic grayling, Thymallus arcticus (Pallas). Mont. 
TABLE 1 (continued)

Smelts, family Osmeridae

Rainbow smelt, Osmerus mordax (Mitchill). N J.

Mudminnows, family Umbridae

Olympic mudminnow, Novumbra hubbsi Schultz. Wash.

Central mudminnow, Umbra limi (Kirtland). S Dak, Vt.

Pikes, family Esocidae

Northern muskellunge, Esox masquinongy masquinongy Mitchill. Vt.

Ohio muskellunge, Esox masquinongy ohiensis Kirtland. Ind, $\mathrm{Ky}$, Ohio.

Minnows, family Cyprinidae

Stoneroller, Campostoma anomalum (Rafinesque). La.

Mexican stoneroller, Campostoma ornatum Girard. Ariz, Tex.

Redside dace, Clinostomus elongatus (Kirtland). Mich.

Rosyside dace, Clinostomus funduloides Girard. Del, Md.

Lake chub, Couesius plumbeus (Agassiz). Mass.

Devils River minnow, Dionda diaboli Hubbs and Brown. Tex.

Ozark minnow, Dionda nubila (Forbes). Wisc.

Desert dace, Eremichthys acros Hubbs and Miller. Nev.

Western tonguetied minnow, Exoglossum laurae hubbsi (Trautman). Ohio.

Eastern tonguetied minnow, Exoglossum laurae laurae (Hubbs). W Va.

Cutlips minnow, Exoglossum maxillingua (Lesueur). Del, Md, Vt, W Va.

Leatherside chub, Gila copei (Jordan and Gilbert). Wyo.

Thicktail chub, Gila crassicauda (Baird and Girard). Calif.

* Humpback chub, Gila cypha Miller. Ariz, Colo, Utah, Wyo.

Bonytail, Gila elegans Baird and Girard. Calif, Nev, Utah.

* Mohave chub, Gila mohavensis (Snyder). Calif.

Roundtail chub, Gila robusta Baird and Girard. N Mex.

*Pahranagat chub, Gila robusta jordani Tanner. Nev.

Flame chub, Hemitremia flammea (Jordan and Gilbert). Tenn.

California roach, Hesperoleucus symmetricus (Baird and Girard). Ore.

Brassy minnow, Hybognathus hankinsoni Hubbs. Mo.

Cypress minnow, Hybognathus hayi Jordan. Mo.

Bigeye chub, Hybopsis amblops (Rafinesque). Mich.

Slender chub, Hybopsis cahni Hubbs and Crowe. Tenn.

Sturgeon chub, Hybopsis gelida (Girard). Mo, Mont, Neb, S Dak, Wyo.

Flathead chub, Hybopsis gracilis (Richardson). S Dak.

Sicklefin chub, Hybopsis meeki Jordan and Evermann. Mo, S Dak.

Spotfin chub, Hybopsis monacha (Cope). Tenn.

Silver chub, Hybopsis storeriana (Kirtland). Mich, S Dak.
Gravel chub, Hybopsis $x$-punctata Hubbs and Crowe. Wisc.

Least chub, lotichthys phlegethontis (Cope). Utah.

White River spinedace, Lepidomeda albivallis Miller and Hubbs. Nev.

Iittle Colorado spinedace, Lepidomeda vittata Cope. Ariz.

Spikedace, Meda fulgida Girard. Ariz.

* Moapa dace, Moapa coriacea Hubbs and Miller. Nev.

Hornyhead chub, Nocomis biguttatus (Kirtland). Neb, S Dak, Wyo.

Pallid shiner, Notropis amnis Hubbs and Greene. Mo.

Comely shiner, Notropis amoenus (Abbott). Md, N Y.

Pugnose shiner, Notropis anogenus Forbes. Wisc.

Popeye shiner, Notropis ariommus (Cope). W Va.

Emerald shiner, Notropis atherinoides Rafinesque. Mass.

Blackspot shiner, Notropis atrocaudalis Evermann. La.

Bridle shiner, Notropis bifrenatus (Cope). Md, Penn.

River shiner, Notropis blennius (Girard). S Dak, W Va.

Bigeye shiner, Notropis boops Gilbert. La.

Ghost shiner, Notropis buchanani Meek. W Va.

Bluntface shiner, Notropis camurus (Jordan and Meek). La.

Ironcolor shiner, Notropis chalybaeus (Cope). Del, Md, Mich, Wisc.

Chihuahua shiner, Notropis chihuahua Woolman. Tex.

Pugnose minnow, Notropis emiliae (Hay). Mo.

Blackchin shiner, Notropis heterodon (Cope). N Dak, Penn, S Dak.

Blacknose shiner, Notropis helerolepis Eigenmann and Eigenmann. Mo, Neb, Penn, S Dak.

Red shiner, Notropis lutrensis (Baird and Girard). Wisc.

Cape Fear shiner, Notropis mekistocholas Snelson (1971). N Car.

Colorless shiner, Notropis perpallidus Hubbs and Black. La.

Silver shiner, Notropis photogenis (Cope). Mich.

Proserpine shiner, Notropis proserpinus (Girard). Tex.

Rosyface shiner, Notropis rubellus (Agassiz). S Dak.

Sabine shiner, Notropis sabinae Jordan and Gilbert. Mo.

New River shiner, Notropis scabriceps (Cope). W Va.

Roughhead shiner, Notropis semperasper Gilbert Va.

Silverband shiner, Notropis shumardi (Girard). S Dak.

Topeka shiner, Notropis topeka Gilbert. Neb, S Dak.

Redfin shiner, Notropis umbratilis (Girard). Wisc.

Skygazer shiner, Notropis uranoscopus Suttkus. Ala.

Mimic shiner, Notropis volucellus (Cope). Wisc.

Bluenose shiner, Notropis welaka Evermann and Kendall. Fla, La.

Steelcolor shiner, Notropis whipplei (Girard). La. Cahaba shiner, Notropis sp. Ala. 
TABLE 1 (continued)

Minnows, family Cyprinidae (continued)

Suckermouth minnow, Phenacobius mirabilis (Girard). La, S Dak, W Va, Wyo.

Kanawha minnow, Phenacobius teretulus Cope. W Va.

Northern redbelly dace, Phoxinus eos (Cope). Mass, Neb, S Dak.

Southern redbelly dace, Phoxinus erythrogaster (Rafinesque). Penn, W Va.

Finescale dace, Phoxinus neogaeus Cope. Mont, Neb, S Dak, Wyo.

Mountain redbelly dace, Phoxinus oreas (Cope). W Va.

Bluntnose minnow, Pimephales notatus (Rafinesque). Conn, La, Neb.

Eastern slim minnow, Pimephales tenellus parviceps (Hubbs and Black). Mo.

*Woundfin, Plagopterus argentissimus Cope. Ariz, Nev, Utah.

* Colorado squawfish, Ptychocheilus lucius Girard. Ariz, Calif, Colo, Nev, Utah.

Blacknose dace, Rhinichthys atratulus (Hermann). Neb, S Dak.

Longnose dace, Rhinichthys cataractae (Valenciennes). Del, Ia.

*Kendall Warm Springs dace, Rhinichthys osculus thermalis Hubbs and Kuehne. Wyo.

Owens speckled dace, Rhinichthys osculus subsp. Calif.

Millicoma dace, Rhinichthys sp. Ore.

Pearl dace, Semotilus margarita (Cope). Conn, Md, Neb, S Dak, W Va, Wyo.

Loach minnow, Tiaroga cobitis Girard. Ariz.

Suckers, family Catostomidae

Quillback, Carpiodes cyprinus (Lesueur). Md, Vt.

Highfin carpsucker, Carpiodes velifer (Rafinesque). Neb.

Longnose sucker, Catostomus catostomus (Forster). Md, Mass, Penn, S Dak, W Va.

Modoc sucker, Catostomus microps Rutter. Calif.

Warner sucker, Catostomus warnerensis Snyder. Ore.

Shortnose sucker, Chasmistes brevirostris Cope. Calif, Ore.

*Cui-ui, Chasmistes cujus Cope. Nev.

Blue sucker, Cycleptus elongatus (Lesueur). Ind, Mont, N Dak, S Dak, W Va, Wisc.

Lost River sucker, Deltistes luxatus (Cope). Calif.

Creek chubsucker, Erimyzon oblongus (Mitchill). Md, Wisc.

Lake chubsucker, Erimyzon sucetta (Lacépède). Wisc.

Northern hog sucker, Hypentelium nigricans (Lesueur). S Dak.

Black buffalo, Ictiobus niger (Rafinesque). Mich, Neb.

Spotted sucker, Minytrema melanops (Rafinesque). Md.

Bigeye jumprock, Moxostoma ariommum Robins and Raney. $\mathrm{Va}$.

River redhorse, Moxostoma carinatum (Cope). Fla, Kan, La, Mich, W Va.

Black redhorse, Moxostoma duquesnei (Lesueur). W Va.

Golden redhorse, Moxostoma erythrurum (Rafinesque). Neb, S Dak.
Rustyside sucker, Moxostoma hamiltoni (Raney and Lachner). Va.

Greater redhorse, Moxostoma valenciennesi Jordan. Wisc.

Humpback sucker, Xyrauchen texanus (Abbott). Calif, Utah.

Freshwater catfishes, family Ictaluridae

Blue catfish, lctalurus furcatus (Lesueur). Neb, S Dak.

Black bullhead, Ictalurus melas (Rafinesque). Vt.

Yellow bullhead, Ictalurus natalis (Lesueur). S Dak.

Brown bullhead, Ictalurus nebulosus (Lesueur). La, Mo, S Dak.

Smoky madtom, Noturus baileyi Taylor. Tenn.

Mountain madtom, Noturus eleutherus Jordan. Penn.

Slender madtom, Noturus exilis Nelson. Wisc.

Yellowfin madtom, Noturus flavipinnis Taylor. Ga, Tenn, Va.

Orangefin madtom, Noturus gilberti Jordan and Evermann. Va.

Tadpole madtom, Noturus gyrinus (Mitchill). Md.

Margined madtom, Noturus insignis (Richardson). Del.

Frecklebelly madtom, Noturus munitus Suttkus and Taylor. Ala, Miss.

Neosho madtom, Noturus placidus Taylor. Kan, Mo.

Northern madtom, Noturus stigmosus Taylor. Mich.

Flathead catfish, Pylodictis olivaris (Rafinesque). Mich, N Dak.

Widemouth blindcat, Satan eurystomus Hubbs and Bailey. Tex.

Toothless blindcat, Trogloglanis pattersoni Eigenmann. Tex.

Cavefishes, family Amblyopsidae

Ozark cavefish, Amblyopsis rosae (Eigenmann). Ark, Mo.

Northern cavefish, Amblyopsis spelaea DeKay. Ind.

Spring cavefish, Chologaster agassizi Putnam. Ill.

Southern cavefish, Typhlichthys subterraneus Girard. Ind.

Pirate perches, family Aphredoderidae

Pirate perch, Aphredoderus sayanus (Gilliams). N Y, Penn.

Trout-perches, family Percopsidae

Trout-perch, Percopsis omiscomaycus (Walbaum). Conn, Ky, Mass, Mont, N Dak, S Dak.

Codfishes, family Gadidae

Burbot, Lota lota (Linnaeus). Conn, Mass, Mo, Ohio.

Atlantic tomcod, Microgadus tomcod (Walbaum). N J.

Killifishes, family Cyprinodontidae

White River springfish, Crenichthys baileyi (Gilbert). Nev.

Railroad Valley springfish, Crenichthys nevadae Hubbs. Nev.

*Devils Hole pupfish, Cyprinodon diabolis Wales. Nev.

*Comanche Springs pupfish, Cyprinodon elegans Baird and Girard. Tex. 
Table 1 (continued)

Killifishes, family Cyprinodontidae (continued)

Desert pupfish, Cyprinodon macularius Baird and Girard. Ariz, Calif.

*Tecopa pupfish, Cyprinodon nevadensis calidae Miller. Calif.

Ash Meadows pupfish, Cyprinodon nevadensis mionectes Miller. Nev.

* Warm spring pupfish, Cyprinodon nevadensis pectoralis Miller. Nev.

Shoshone pupfish, Cyprinodon nevadensis shoshone Miller. Calif.

*Owens pupfish, Cyprinodon radiosus Miller. Calif.

*Pahrump killifish, Empetrichthys latos Miller. Nev.

Golden topminnow, Fundulus chrysotus (Günther). Mo.

Western banded killifish, Fundulus diaphanus menona Jordan and Copeland. Penn, S Dak.

Plains killifish, Fundulus kansae Garman. Mo, Mont.

Spotfin killifish, Fundulus luciae (Baird). Del.

Starhead topminnow, Fundulus notti (Agassiz). Mich, Mo, Wisc.

Plains topminnow, Fundulus sciadicus Cope. Kan, S Dak.

Waccamaw killifish, Fundulus waccamensis Hubbs and Raney. N Car.

Barrens topminnow, Fundulus sp. Tenn.

Livebearers, family Poeciliidae

*Big Bend gambusia, Gambusia gaigei Hubbs. Tex.

San Marcos gambusia, Gambusia georgei Hubbs and Peden. Tex.

* Clear Creek gambusia, Gambusia heterochir Hubbs. Tex.

*Pecos gambusia, Gambusia nobilis (Baird and Girard). N Mex, Tex.

Blotched gambusia, Gambusia senilis Girard. Tex.

Amazon molly, Poecilia formosa (Girard). Tex.

* Gila topminnow, Poeciliopsis occidentalis (Baird and Girard). Ariz.

Silversides, family Atherinidae

Brook silverside, Labidesthes sicculus (Cope). Md, Penn.

Mississippi silverside, Menidia audens Hay. Mo.

Key silverside, Menidia conchorum Hildebrand and Ginsburg. Fla.

Waccamaw silverside, Menidia extensa Hubbs and Raney. N Car.

Sticklebacks, family Gasterosteidae

Fourspine stickleback, Apeltes quadracus (Mitchill). Mass, Penn.

Brook stickleback, Culaea inconstans (Kirtland). Mass, N Mex, Penn, Vt.

Threespine stickleback, Gasterosteus aculeatus Linnaeus. Mass.

*Unarmored threespine stickleback, Gasterosteus aculeatus williamsoni Girard. Calif.

Blackspotted stickleback, Gasterosteus wheatlandi Putnam. N Y.

Black stickleback, Gasterosteus sp. Wash.

Ninespine stickleback, Pungitius pungitius (Linnaeus). Mass.

Sunfishes, family Centrarchidae

Mud sunfish, Acantharchus pomotis (Baird). Del, Md.
Sacramento perch, Archoplites interruptus (Girard). Calif.

Banded pygmy sunfish, Elassoma zonatum Jordan. Ill.

Blackbanded sunfish, Enneacanthus chaetodon (Baird). Del, Md.

Bluespotted sunfish, Enneacanthus gloriosus (Holbrook). Del, N Y.

Banded sunfish, Enneacanthus obesus (Girard). Del, N. Y.

Pumpkinseed, Lepomis gibbosus (Linnaeus). Mo.

Warmouth, Lepomis gulosus (Cuvier). Md, Penn.

Bantam sunfish, Lepomis symmetricus Forbes. Ill, Mo.

Suwannee bass, Micropterus notius Bailey and Hubbs. Fla.

Perches, family Percidae

Crystal darter, Ammocrypta asprella (Jordan). Ala, Miss, Wisc.

Western sand darter, Ammocrypta clara Jordan and Meek. Ia, Mo.

Eastern sand darter, Ammocrypta pellucida (Putnam). Mich, Ohio, Penn.

Scaly sand darter, Ammocrypta vivax Hay. Ky, Mo.

Sharphead darter, Etheostoma acuticeps Bailey. Tenn.

Mud darter, Etheostoma asprigene (Forbes). Ky, Wisc.

Rainbow darter, Etheostoma caeruleum Storer. La.

Bluebreast darter, Etheostoma camurum (Cope). Ill.

Bluntnose darter, Etheostoma chlorosomum (Hay). Wisc.

Ashy darter, Etheostoma cinereum Storer. Ky.

Arkansas darter, Etheostoma cragini Gilbert. Kan, Mo, Okla.

Coldwater darter, Etheostoma ditrema Ramsey and Suttkus. Ala, Ga, Tenn.

* Fountain darter, Etheostoma fonticola (Jordan and Gilbert). Tex.

Swamp darter, Etheostoma fusiforme (Girard). Conn, Mass, Penn.

Rio Grande darter, Etheostoma grahami (Girard). Tex.

Harlequin darter, Etheostoma histrio Jordan and Gilbert. Ill, Ky, Mo.

Least darter, Etheostoma microperca Jordan and Gilbert. Kan, Penn.

Yellowcheek darter, Etheostoma moorei Raney and Suttkus. Ark.

Niangua darter, Etheostoma nianguae Gilbert and Meek. Mo.

*Watercress darter, Etheostoma nuchale Howell and Caldwell. Ala.

Okaloosa darter, Etheostoma okaloosae (Fowler). Fla.

Waccamaw darter, Etheostoma perlongum (Hubbs and Raney). N Car.

Stippled darter, Etheostoma punctulatum (Agassiz). Kan.

Bayou darter, Etheostoma rubrum Raney and Suttkus. Miss.

* Maryland darter, Etheostoma sellare (Radcliffe and Welsh). Md.

Speckled darter, Etheostoma stigmaeum (Jordan). Kan. 
TABLE 1 (continued)
Perches, family Percidae (continued)
Tippecanoe darter, Etheostoma tippecanoe Jordan and Evermann. Ky, Penn, Tenn, W Va.
Trispot darter, Etheostoma trisella Bailey and Richards. Ala, Ga, Tenn.
Tuscumbia darter, Etheostoma tuscumbia Gilbert and Swain. Ala, Tenn.
Glassy darter, Etheostoma vitreum (Cope). Md.
Redfin darter, Etheostoma whipplei (Girard). Mo.
Unnamed darter, Etheostoma sp. Ala.
Duck River darter, Etheostoma sp. Tenn.
Goldline darter, Percina aurolineata Suttkus and Ramsey. Ala, Ga.
Blotchside logperch, Percina burtoni Fowler. Tenn, Va.
Channel darter, Percina copelandi (Jordan). Ky, W Va.
Bluestripe darter, Percina cymatotaenia (Gilbert and Meek). Mo.
Gilt darter, Percina evides (Jordan and Copeland). Ky.
Freckled darter, Percina lenticula Richards and Knapp. Ala, Ga, La, Miss.
Longhead darter, Percina macrocephala (Cope). Ky, Penn.
Blackside darter, Percina maculata (Girard). Kan, S Dak.
Longnose darter, Percina nasuta (Bailey). Mo.
Leopard darter, Percina pantherina (Moore and Reeves). Okla.
Slenderhead darter, Percina phoxocephala (Nel- son). Ky, Penn, S Dak.
Roanoke logperch, Percina rex (Jordan and Ever- mann). Va.

River darter, Percina shumardi (Girard). Ky, Mich.

Olive darter, Percina squamata (Gilbert and Swain). Ky.

Stargazing darter, Percina uranidia (Jordan and Gilbert). Ky.

Narrowsaddle darter, Percina sp. Ga, Tenn.

Sauger, Stizostedion canadense (Smith). Ky, Ohio, W Va.

* Blue pike, Stizostedion vitreum glaucum Hubbs. N Y, Ohio, Penn.

Walleye, Stizostedion vitreum vitreum (Mitchill). Ky.

Gobies, family Gobiidae

O'opu nakea, Awaous stamineus (Eydoux and Souleyet). Hawaii.

- Lentipes concolor (Gill). Hawaii.

__L Lentipes seminudus Günther. Hawaii.

O'opu nopili, Sicydium stimpsoni Gill. Hawaii.

Sculpins, family Cottidae

Rough sculpin, Cottus asperrimus Rutter. Calif.

Slimy sculpin, Cottus cognatus Richardson. N J.

Shorthead sculpin, Cottus confusus Bailey and Bond. Mont.

Bear Lake sculpin, Cottus extensus Bailey and Bond. Ida, Utah.

Shoshone sculpin, Cottus greenei (Gilbert and Culver). Ida.

Pit sculpin, Cottus pitensis Bailey and Bond. Calif, Ore.

Pygmy sculpin, Cottus pygmaeus Williams. Ala.

Spoonhead sculpin, Cottus ricei (Nelson). Mich, Mont, Ohio.

\section{STATE LISTS}

In this section is a list of the fishes for each state, by common name only, that are regarded as threatened, followed by their status as defined previously.

\section{Alabama}

Lake sturgeon, depleted (possibly extinct).

Atlantic sturgeon, depleted.

Shovelnose sturgeon, depleted.

Skygazer shiner, rare.

Cahaba shiner, rare (possibly endangered).

Frecklebelly madtom, depleted.

Crystal darter, depleted.

Coldwater darter, depleted.

Watercress darter, rare and endangered.

Trispot darter, rare (possibly extinct).

Tuscumbia darter, depleted.

Unnamed darter (Cypress Cr.), rare (possibly endangered)

Goldline darter, rare (possibly endangered).

Freckled darter, depleted.

Pygmy sculpin, rare and endangered.

$$
\text { Alaska }
$$

Angayukaksurak char (N. slope, Brooks Range), rare.

\section{Arizona}

Arizona trout (White Mts.), rare (protected).

Mexican stoneroller, rare.

Humpback chub, rare and endangered.

Little Colorado spinedace, rare.

Spikedace, rare (possibly endangered).

Woundfin, rare and endangered.

Loach minnow, rare and endangered.

Desert pupfish, rare.

Gila topminnow, rare and endangered (under propagation).

\section{Arkansas}

Ozark cavefish, rare.

Yellowcheek darter, rare.

\section{California}

Those species marked by an asterisk are fully protected by provisions of the State Fish and Game Code enacted in 1970.

Lahontan cutthroat trout, depleted.

*Piute cutthroat trout, depleted.

Little Kern golden trout, depleted.

Redband trout (a few streams of McCloud R. basin), rare.

*Thicktail chub, indeterminate (possibly extinct). Bonytail, rare.

*Mohave chub, endangered. 
*Colorado squawfish, endangered.

Owens speckled dace, indeterminate.

*Modoc sucker, indeterminate.

* Shortnose sucker, rare.

* Lost River sucker, rare.

*Humpback sucker, rare.

Tecopa pupfish, endangered (possibly extinct).

Shoshone pupfish, endangered (possibly extinct).

* Owens pupfish, endangered (Miller and Pister, 1971).

Desert pupfish, depleted.

"Unarmored threespine stickleback, endangered.

Sacramento perch, depleted.

Rough sculpin, indeterminate.

Pit sculpin, indeterminate.

\section{Colorado}

Colorado River cutthroat trout, rare and endangered. Greenback cutthroat trout, rare and endangered. Rio Grande cutthroat trout, rare and endangered.

Humpback chub, rare and endangered.

Colorado squawfish, rare.

\section{Connecticut}

American brook lamprey, rare.

Shortnose sturgeon, endangered.

Atlantic sturgeon, endangered.

Round whitefish (East Twin L., possibly intro. duced), endangered.

Atlantic salmon, endangered.

Bluntnose minnow, rare.

Pearl dace, rare.

Trout-perch, rare.

Burbot, endangered.

Swamp darter, rare.

\section{Delaware}

Shortnose sturgeon, endangered.

Atlantic sturgeon, depleted.

Longnose gar, endangered (possibly extinct).

American shad, depleted.

Rosyside dace, rare.

Cutlips minnow, rare.

Ironcolor shiner, endangered (possibly extinct).

Longnose dace, rare.

Margined madtom, rare.

Spotfin killifish, rare.

Mud sunfish, endangered.

Blackbanded sunfish, indeterminate.

Bluespotted sunfish, rare.

Banded sunfish, indeterminate.

\section{Florida}

Bluenose shiner, rare.

River redhorse, endangered.

Key silverside, rare and endangered.

Suwannee bass, rare.

Okaloosa darter, rare (possibly endangered).

\section{Georgia}

Lake sturgeon, depleted.

Atlantic sturgeon, depleted.

Yellowfin madtom, rare (possibly extinct).

Coldwater darter, depleted.

Trispot darter, rare.

Goldline darter, rare (possibly endangered) .

Freckled darter, depleted.

Narrowsaddle darter (Conasauga R. of Coosa R. drainage), rare.

\section{Hawaii}

O'opu nakea, depleted (on Oahu).

Lentipes concolor, rare and endangered.

Lentipes seminudus, rare (extinct on Oahu).

O'opu nopili, rare (on Oahu).

\section{Idaho}

Fishing is permitted for sturgeon, but all must be released.

White sturgeon, rare and endangered.

Bear Lake whitefish, rare.

Bonneville cisco, rare.

Bonneville whitefish, rare.

Bear Lake sculpin, rare.

Shoshone sculpin, rare.

\section{Illinois}

All of the fishes listed are protected in the Illinois Game and Fish Codes.

Spring cavefish, rare.

Banded pygmy sunfish, rare.

Bantam sunfish, rare.

Bluebreast darter, rare.

Harlequin darter, rare.

\section{Indiana}

All of these fishes are protected from harvest of any kind.

Ohio River muskellunge, endangered.

Blue sucker, rare.

Northern cavefish, endangered.

Southern cavefish, endangered.

\section{lowa}

Both sturgeons are protected by State or Federal regulations.

Lake sturgeon, rare and endangered.

Pallid sturgeon, rare and endangered.

Blacknose shiner, rare.

Longnose dace, rare.

Western sand darter, rare.

\section{Kansas}

Present evidence indicates that neither the bigeye chub nor the blacknose shiner still exist in Kansas.

Chestnut lamprey, rare. 
Pallid sturgeon, rare.

River redhorse, depleted.

Neosho madtom, endangered.

Plains topminnow, rare.

Arkansas darter, depleted.

Least darter, rare.

Stippled darter, rare.

Speckled darter, rare.

Blackside darter, rare.

\section{Kentucky}

Pallid sturgeon, endangered.

Shovelnose sturgeon, endangered.

Ohio muskellunge, rare.

Trout-perch, endangered.

Scaly sand darter, rare.

Mud darter, rare and endangered.

Ashy darter, rare.

Harlequin darter, rare and endangered.

Tippecanoe darter, endangered.

Channel darter, rare.

Gilt darter, rare.

Longhead darter, endangered.

Slenderhead darter, rare.

River darter, rare.

Olive darter, rare.

Stargazing darter, rare.

Sauger, depleted.

Walleye, depleted.

\section{Louisiana}

Pallid sturgeon, rare.

Stoneroller, rare.

Blackspot shiner, rare.

Bigeye shiner, rare.

Bluntface shiner, rare.

Colorless shiner, rare.

Bluenose shiner, rare.

Steelcolor shiner, rare.

Suckermouth minnow, rare.

Bluntnose minnow, rare.

River redhorse, rare.

Rainbow darter (undescribed form from tributaries on E. side Miss. R.), rare.

Freckled darter, rare and endangered.

\section{Maine}

Atlantic sturgeon, rare (possibly extinct).

Atlantic salmon, rare and endangered (sea-run form).

Sunapee trout, rare and endangered.

Blueback trout, rare.

\section{Maryland}

Ohio lamprey, rare and depleted.

Shortnose sturgeon, rare.

Atlantic sturgeon, depleted.

Paddlefish, rare and depleted.

Bowfin, rare and endangered.
Rosyside dace, rare.

Cutlips minnow, depleted.

Comely shiner, depleted.

Bridle shiner, rare and depleted.

Ironcolor shiner, rare and depleted.

Pearl dace, rare and depleted.

Quillback, depleted.

Longnose sucker, rare and endangered.

Creek chubsucker, depleted.

Spotted sucker, rare and endangered.

Tadpole madtom, rare and depleted.

Brook silverside, rare.

Mud sunfish, rare and endangered.

Blackbanded sunfish, rare and endangered.

Warmouth, depleted.

Maryland darter, rare and endangered.

Glassy darter, rare and endangered.

\section{Massachusetts}

American brook lamprey, rare.

Shortnose sturgeon, rare and endangered.

Atlantic sturgeon, rare and endangered.

Atlantic salmon, rare and endangered.

Lake chub, rare and depleted.

Emerald shiner, rare and depleted.

Northern redbelly dace, rare and depleted.

Longnose sucker, rare.

Trout-perch, rare and endangered.

Burbot, rare and depleted.

Fourspine stickleback, rare.

Brook stickleback, rare.

Threespine stickleback, rare.

Ninespine stickleback, rare.

Swamp darter, rare.

\section{Michigan}

Lake sturgeon, rare and endangered.

Mooneye, rare.

Longjaw cisco, almost extinct.

Kiyi, rare and endangered.

Shortjaw cisco, rare and endangered.

Redside dace, rare.

Bigeye chub, rare.

Silver chub, rare.

Ironcolor shiner, rare.

Silver shiner, rare.

Black buffalo, rare.

River redhorse, rare.

Northern madtom, rare.

Flathead catfish, rare.

Starhead topminnow, depleted.

Eastern sand darter, rare.

River darter, rare.

Spoonhead sculpin, rare and depleted.

\section{Minnesota}

All of the following are legally protected by closed season or by size limit and/or bag limit. 
Lake sturgeon, rare.

Shovelnose sturgeon, rare.

Paddlefish, rare.

\section{Mississippi}

Atlantic sturgeon, depleted.

Shovelnose sturgeon, depleted.

Frecklebelly madtom, depleted.

Crystal darter, depleted.

Bayou darter, rare (possibly endangered).

Freckled darter, depleted.

\section{Missouri}

Southern brook lamprey, rare.

American brook lamprey, rare.

Lake sturgeon, endangered or depleted.

Pallid sturgeon, endangered or depleted.

Alligator gar, rare.

Alabama shad, rare.

Brassy minnow, rare.

Cypress minnow, endangered or extinct.

Sturgeon chub, rare or endangered.

Sicklefin chub, rare or endangered.

Pallid shiner, endangered or extinct.

Pugnose minnow, rare (possibly endangered).

Blacknose shiner, depleted.

Sabine shiner, rare.

Eastern slim minnow, rare.

Brown bullhead, rare.

Neosho madtom, rare or endangered.

Ozark cavefish, rare.

Burbot, rare.

Golden topminnow, endangered or extinct.

Plains killifish, rare.

Starhead topminnow, depleted.

Mississippi silverside, rare.

Pumpkinseed, rare.

Bantam sunfish, rare.

Western sand darter, depleted.

Scaly sand darter, depleted.

Arkansas darter, rare.

Harlequin darter, rare (possibly endangered).

Niangua darter, rare.

Redfin darter, endangered or extinct.

Bluestripe darter, rare.

Longnose darter, rare (possibly endangered).

\section{Montana}

White sturgeon, rare.

Pallid sturgeon, rare.

Shortnose gar, rare.

Arctic grayling, rare.

Westslope cutthroat trout, endangered.

Eastslope cutthroat trout, endangered.

Sturgeon chub, rare.

Finescale dace, rare.

Blue sucker, rare.

Trout-perch, rare.

Plains killifish, rare.
Shorthead sculpin, rare.

Spoonhead sculpin, rare.

\section{Nebraska}

Chestnut lamprey, endangered.

Silver lamprey, endangered.

Lake sturgeon, endangered.

Pallid sturgeon, endangered.

Mooneye, endangered.

Sturgeon chub, endangered (possibly extinct).

Hornyhead chub, rare.

Blacknose shiner, rare

Topeka shiner, endangered.

Northern redbelly dace, rare and endangered.

Finescale dace, endangered.

Bluntnose minnow, endangered (possibly extinct).

Blacknose dace, endangered.

Pearl dace, rare.

Highfin carpsucker, endangered (possibly extinct) .

Black buffalo, indeterminate.

Golden redhorse, rare.

Blue catfish, indeterminate.

\section{Nevada}

All of the following except the trout and woundfin are legally protected although the Indians of Pyramid Lake utilize the cui-ui for food. Pure stocks of the Lahontan cutthroat trout are scarce, but it is propagated as a game fish; the White River springfish is in a protected refuge near Sunnyside.

Snake Valley cutthroat trout (Spring Valley), rare.

Desert dace, rare.

Bonytail, endangered.

Pahranagat roundtail chub, rare and endangered.

White River spinedace, rare.

Moapa dace, rare and endangered.

Woundfin, rare and endangered.

Colorado squawfish, rare and endangered.

Cui-ui, rare and endangered.

White River springfish, rare.

Railroad Valley springfish, rare.

Devils Hole pupfish, rare and endangered.

Ash Meadows pupfish, endangered.

Warm Spring pupfish, rare and endangered.

Pahrump killifish, rare and endangered.

\section{New Hampshire}

Atlantic sturgeon, rare.

Atlantic salmon, rare (sea-run form). Sunapee trout, endangered.

\section{New Jersey}

Shortnose sturgeon, endangered.

Atlantic sturgeon, rare.

American shad, rare.

Rainbow smelt, endangered.

Atlantic tomcod, indeterminate.

Slimy sculpin, indeterminate. 


\section{New Mexico}

Rio Grande cutthroat trout, rare and endangered. Gila trout, rare and endangered.

Roundtail chub, endangered (perhaps extinct in Gila R. basin).

Pecos gambusia, endangered.

Brook stickleback, rare (relict).

\section{New York}

Shortnose sturgeon, endangered.

Lake sturgeon, rare.

American shad, indeterminate.

Lake whitefish, indeterminate.

Round whitefish, rare.

Atlantic salmon, rare (landlocked form).

Comely shiner, probably depleted in Hudson River drainage.

Pirate perch, endangered.

Blackspotted stickleback, endangered.

Bluespotted sunfish, rare.

Banded sunfish, rare.

Blue pike, endangered.

\section{North Carolina}

Cape Fear shiner, rare.

Waccamaw killifish, rare.

Waccamaw silverside, rare.

Waccamaw darter, rare.

\section{North Dakota}

Pallid sturgeon, rare.

Blackchin shiner, rare.

Blue sucker, endangered.

Flathead catfish, rare.

Trout-perch, rare.

\section{Ohio}

Lake sturgeon, endangered.

Mooneye, rare.

Cisco or lake herring, rare.

Lake whitefish, rare.

Brook trout, rare.

Lake trout, endangered.

Great Lakes muskellunge, rare.

Western tonguetied minnow, endangered.

Burbot, rare.

Eastern sand darter, depleted.

Sauger, rare.

Blue pike, endangered (commercially extinct).

Spoonhead sculpin, depleted.

\section{Oklahoma}

Shovelnose sturgeon, rare and endangered.

Arkansas darter, rare and endangered.

Leopard darter, rare and endangered.

\section{Oregon}

Pit-Klamath brook lamprey, rare.

Alvord cutthroat trout, rare.

Redband trout, rare.

Dolly Varden, rare (only in Klamath basin).

Cahifornia roach, rare.

Millicoma dace (restricted to Coos R. system), rare.

Warner sucker, rare.

Shortnose sucker, rare.

Pit sculpin, rare (possibly extinct).

\section{Pennsylvania}

Allegheny brook lamprey, rare.

Shortnose sturgeon, endangered.

Lake sturgeon, endangered.

Atlantic sturgeon, endangered.

Paddlefish, endangered.

Bowfin, depleted.

Cisco or lake herring, rare.

Lake whitefish, rare.

Bridle shiner, rare.

Blackchin shiner, endangered.

Blacknose shiner, endangered.

Southern redbelly dace, depleted.

Longnose sucker, endangered.

Mountain madtom, endangered.

Pirate perch, endangered (possibly extinct).

Western banded killifish, depleted.

Brook silverside, rare.

Fourspine stickleback, endangered.

Brook stickleback, rare.

Warmouth, rare.

Eastern sand darter, endangered.

Swamp darter, endangered (possibly extinct).

Least darter, endangered.

Tippecanoe darter, endangered.

Longhead darter, endangered.

Slenderhead darter, endangered.

Blue pike, endangered.

\section{South Carolina}

Atlantic sturgeon, depleted.

\section{South Dakota}

Silver lamprey, endangered.

Lake sturgeon, endangered.

Pallid sturgeon, rare.

Shovelnose sturgeon, depleted.

Longnose gar, rare.

Bowfin, rare.

American eel, endangered.

Skipjack herring, rare.

Mooneye, endangered.

Central mudminnow, rare.

Sturgeon chub, rare.

Flathead chub, rare. 
Sicklefin chub, rare.

Silver chub, rare.

Hornyhead chub, rare.

River shiner, rare.

Blackchin shiner, endangered.

Blacknose shiner, endangered.

Rosyface shiner, rare.

Silverband shiner, rare.

Topeka shiner, rare.

Suckermouth minnow, endangered (possibly extinct).

Northern redbelly dace, rare.

Finescale dace, rare.

Blacknose dace, rare.

Pearl dace, endangered.

Longnose sucker, rare.

Blue sucker, depleted.

Northern hog sucker, rare.

Golden redhorse, rare.

Blue catfish, rare.

Yellow bullhead, rare.

Brown bullhead, rare.

Trout-perch, rare.

Banded killifish, rare.

Plains topminnow, rare.

Blackside darter, rare.

Slenderhead darter, rare.

\section{Tennessee}

Flame dace, depleted.

Slender chub, rare and endangered.

Spotfin chub, endangered.

Smoky madtom, rare (possibly extinct).

Yellowfin madtom, rare and endangered (see Taylor et al., 1971).

Barrens topminnow (Duck and Cumberland R. drainages), rare.

Sharphead darter, rare (possibly extinct).

Coldwater darter, rare.

Tippecanoe darter, depleted.

Trispot darter, rare.

Tuscumbia darter, endangered (possibly extinct).

Duck River darter, rare.

Blotchside logperch, rare.

Narrowsaddle darter (Conasauga R. of Coosa R. drainage), rare.

\section{Texas}

Mexican stoneroller, rare.

Devils River minnow, rare.

Chihuahua shiner, rare.

Proserpine shiner, rare.

Widemouth blindcat, rare.

Toothless blindcat, rare.

Comanche Springs pupfish, rare and endangered.

Big Bend gambusia, rare.

San Marcos gambusia, rare.

Clear Creek gambusia, rare.

Pecos gambusia, rare and endangered.

Blotched gambusia, rare.

Amazon molly, rare.

Fountain darter, rare.

Rio Grande darter, rare.
Utah

Bear Lake whitefish, rare.

Bonneville cisco, rare.

Bonneville whitefish, rare.

Humpback chub, rare and endangered.

Bonytail, depleted.

Least chub, rare.

Woundfin, rare and endangered.

Colorado squawfish, rare and endangered.

Humpback sucker, rare.

Bear Lake sculpin, rare.

\section{Vermont}

Since the Sunapee trout has not been reported in the state for over 30 years, it is presumed to be extinct.

Lake sturgeon, endangered.

Mooneye, rare.

Atlantic salmon, depleted (landlocked form).

Central mudminnow, rare.

Northern muskellunge, endangered.

Cutlips minnow, rare.

Quillback, rare.

Black bullhead, rare.

Brook stickleback, rare.

\section{Virginia}

Roughhead shiner, rare.

Bigeye jumprock, rare.

Rustyside sucker, rare.

Yellowfin madtom, rare.

Orangefin madtom, rare.

Blotchside logperch, rare.

Roanoke logperch, rare.

\section{Washington}

Olympic mudminnow, rare.

Black stickleback, rare.

\section{W est Virginia}

Silver lamprey, rare.

Lake sturgeon, endangered (possibly extinct).

Shovelnose sturgeon, rare, endangered, and depleted.

Paddlefish, rare, endangered, and depleted.

Mooneye, rare.

Eastern tonguetied minnow, depleted.

Cutlips minnow, depleted.

Popeye shiner, rare, endangered, and depleted.

River shiner, depleted.

Ghost shiner, depleted.

New River shiner, depleted.

Suckermouth minnow, depleted.

Kanawha minnow, rare and depleted.

Southern redbelly dace, rare.

Mountain redbelly dace, depleted.

Pearl dace, rare and depleted. 
Longnose sucker, rare (perhaps extinct).

Blue sucker, rare, endangered, and depleted.

River redhorse, rare.

Black redhorse, depleted.

Tippecanoe darter, rare.

Channel darter, rare.

Sauger, depleted.

\section{Wisconsin}

Paddlefish, rare.

American eel, rare.

Goldeye, rare.

Longjaw cisco, endangered.

Kiyi, rare (Lake Michigan).

Blackfin cisco, rare (Lake Superior; probably extinct in Lake Michigan).

Shortnose cisco, rare (Lake Superior) and endangered (Lake Michigan).

Shortjaw cisco, rare (Lake Michigan).

Ozark minnow, rare.

Gravel chub, endangered.

Pugnose shiner, endangered.

Ironcolor shiner, indeterminate.

Red shiner, rare.

Redfin shiner, depleted.

Mimic shiner, endangered.

Blue sucker, rare.

Creek chubsucker, indeterminate.

Lake chubsucker, rare.

Greater redhorse, indeterminate.

Slender madtom, endangered.

Starhead topminnow, rare.

Crystal darter, indeterminate.

Mud darter, rare.

Bluntnose darter, indeterminate.

\section{Wroming}

Three species, the humpback chub, bonytail, and Colorado squawfish, are now believed to be extinct in the state.

Shovelnose sturgeon, rare and endangered.

Goldeye, rare and endangered.

Colorado River cutthroat trout, rare and endangered. Snake River cutthroat trout, rare.

Leatherside chub, rare and endangered.

Sturgeon chub, rare and endangered.

Hornyhead chub, rare and endangered.

Suckermouth minnow, rare and endangered.

Finescale dace, rare and endangered.

Kendall Warm Springs dace, rare.

Pearl dace, rare and endangered.

\section{ACKNOWLEDGMENTS}

This paper constitutes a report of the Endangered Species Committee, the membership of which has already been listed. Important contributions were also made by the following: Marvin 0. Allum, Charles Backlund, Reeve M. Bailey, Richard E. Bass, George T. Baxter,
George Becker, Robert J. Behnke, Peter L. Berrien, Colton H. Bridges, C. J. D. Brown, Bernard T. Carter, James P. Carter, Robert C. Cashner, Lyle M. Christenson, Russell A. Cookingham, Edwin C. Cooper, Frank B. Cross, Franklin C. Daiber, James E. Deacon, David A. Etnier, R. L. Evans, Glen R. Foster, Bennie J. Foutenot, Jr., Carter R. Gilbert, Leonard C. Halnon, Paul E. Hamer, Robert L. Hanten, Robert W. Harrington, Jr., Donald S. Heintzelman, Dale L. Henegar, Carl L. Hubbs, Robert E. Jenkins, Walter T. Keller, Earl R. Kendle, Clayton Lakes, C. C. Lindsey, John A. Maciolek, James Mayhew, Maurice F. Mettee, Jr., Robert L. Miles, W. L. Minckley, James E. Morrow, John A. Musick, George S. Myers, Arthur E. Newell, Carl E. Parker, William L. Pflieger, A. Bruce Pyle, Edward C. Raney, C. Richard Robins, Donn E. Rosen, James C. Schmulbach, Victor J. Schuler, Frank J. Schwartz, James C. Simpson, Philip W. Smith, Royal D. Suttkus, H. O. Swenson, Michio Takata, Bruce A. Thompson, Thomas J. Trelease, O. L. Wallis, Arthur N. Whitney, Walter R. Whitworth, and Cole W. Wilde.

For financial support of field studies on rare and endangered species $I$ am indebted to the National Park Service, National Science Foundation (GB-4854, 14871), National Wildlife Federation, and the Zoological Society of San Diego.

\section{LITERATURE CITED}

Balley, R. M., J. E. Fitch, E. S. Herald, E. A. Lachner, C. C. Lindsey, C. R. Robins, and W. B. Scott. 1970. A list of common and scientific names of fishes from the United States and Canada. 3rd ed. Amer. Fish. Soc. Spec. Publ. 6: 1-150.

Deacon, J., and S. Bunnell. 1970. Man and pupfish. Cry California 5(2): 14-21.

HubBs, C. L. 1971. Lampetra (Entosphenus) lethophaga, new species, the nonparasitic derivative of the Pacific lamprey. Trans. San Diego Soc. Nat. Hist. 16(6): 125-164.

Hubbs, Clark. 1963. An evaluation of the use of rotenone as a means of "improving" sports fishing in the Concho River, Texas. Copeia 1963(1) : 199-203.

Lachner, E. A., C. R. Robins, and W. R. Courtenay, Jr. 1970. Exotic fishes and other aquatic organisms introduced into North America. Smithsonian Contrib. Zool. 59: 1-29.

Larimore, R. W., and P. W. Smith. 1963. The fishes of Champaign County, Illinois, as affected by 60 years of stream changes. Bull. IIl. Nat. Hist. Surv. 28(2) : 299-382. 
McAllister, D. E. 1970. Rare or endangered Canadian fishes. Can. Field-Nat. 84(1) : 5-8.

McDowall, R. M. 1968. Interactions of the native and alien faunas of New Zealand and the problem of fish introductions. Trans. Amer. Fish. Soc. 97(1) : 1-11.

Miller, R. R. 1961. Man and the changing fish fauna of the American Southwest. Paper Mich. Acad. Sci., Arts, and Letters 46 (1960) : 365-404. 1972. Classification of the trouts of Arizona with the description of a new species, Salmo apache. Copeia $1972(3)$-in press.

, AND E. P. PISTer. 1971. Management of the Owens pupfish, Cyprinodon radiosus, in Mono County, California. Trans. Amer. Fish. Soc. $100(3)$ : $502-507$.

Mills, H. B., W. C. Starrett, and F. C. Bellrose. 1966. Man's effect on the fish and wildlife of the Illinois River. Biol. Notes IIl. Nat. Hist. Surv. 57: 1-24.

Minckley, W. L., and J. E. Deacon. 1968. South- western fishes and the enigma of "endangered species." Science 159(3822): 1424-1432.

Myers, G. S. 1965. Gambusia, the fish destroyer. Trop. Fish Hobbyist 13(5): 31-32, 53-54.

ScotT, W. B. 1963. A review of the changes in the fish fauna of Ontario. Trans. Roy. Can. Inst. $34(2)$ : $111-125$.

SмIтh, P. W. 1971. Illinois streams: a classification based on their fishes and an analysis of factors responsible for disappearance of native species. Biol. Notes Ill. Nat. Hist. Surv. 76: 1-14.

Snelson, F. F., JR. 1971. Notropis mekistocholas, a new herbivorous cyprinid fish endemic to the Cape Fear River Basin, North Carolina. Copeia $1971(3)$ : $449-462$.

Taylor, W. R., R. E. Jenkins, and E. A. Lachner. 1971. Rediscovery and description of the ictalurid catfish, Noturus flavipinnis. Proc. Biol. Soc. Wash. 83(41) : 469-476.

Trautman, M. B. 1957. The fishes of Ohio. Ohio State Univ. Press, Columbus, 683 pp. 